\title{
One pair of hands is not like another: Caudate BOLD response in dogs depends on signal source and canine temperament
}

Having previously used functional MRI to map the response to a reward signal in the ventral caudate in awake unrestrained dogs, here we examined the importance of signal source to canine caudate activation. Hand signals representing either incipient reward or no reward were presented by a familiar human (each dog's respective handler), an unfamiliar human, and via illustrated images of hands on a computer screen to 13 dogs undergoing voluntary fMRI. All dogs had received extensive training with the reward and no-reward signals from their handlers and with the computer images and had minimal exposure to the signals from strangers. All dogs showed differentially higher BOLD response in the ventral caudate to the reward versus no reward signals, and there was a robust effect at the group level. Further, differential response to the signal source had a highly significant interaction with a dog's stranger-directed aggression as measured by the C-BARQ canine personality assessment. Relatively "aggressive" dogs showed a higher differential response to the reward signal versus no-reward signal presented by the unfamiliar human, while relatively "calm" dogs showed a higher differential response to the reward signal versus no-reward signal from their handler. This suggests that specific facets of canine temperament bear more strongly on the perceived reward value of relevant communication signals than does reinforcement history, as each of the dogs were reinforced similarly for each signal, regardless of the source (familiar human, unfamiliar human, or computer). A group-level PPI connectivity analysis showed increased functional coupling between the caudate and a region of cortex associated with sensory integration on reward versus no-reward trials. Our findings emphasize the sensitivity of the domestic dog to human social interaction, and may have other implications 
and applications pertinent to the training and assessment of working and pet dogs. 
1 One Pair of Hands Is Not Like Another: Caudate BOLD Response in Dogs

2 Depends on Signal Source and Canine Temperament

3 Peter F. Cook ${ }^{1}$, Mark Spivak ${ }^{2}$, Gregory S. Berns ${ }^{1 *}$

$4 \quad{ }^{1}$ Economics Dept. \& Center for Neuropolicy, Emory University, Atlanta, GA 30322

5 USA

$6 \quad{ }^{2}$ Comprehensive Pet Therapy, Sandy Springs, GA 30328 USA

$7 \quad *$ To whom correspondence should be addressed.

8 Abstract 
9 Having previously used functional MRI to map the response to a reward signal in the 10 ventral caudate in awake unrestrained dogs, here we examined the importance of

11 signal source to canine caudate activation. Hand signals representing either

12 incipient reward or no reward were presented by a familiar human (each dog's

13 respective handler), an unfamiliar human, and via illustrated images of hands on a

14 computer screen to 13 dogs undergoing voluntary fMRI. All dogs had received

15 extensive training with the reward and no-reward signals from their handlers and

16 with the computer images and had minimal exposure to the signals from strangers.

17 All dogs showed differentially higher BOLD response in the ventral caudate to the

18 reward versus no reward signals, and there was a robust effect at the group level.

19 Further, differential response to the signal source had a highly significant interaction

20 with a dog's general aggressivity as measured by the C-BARQ canine personality

21 assessment. Dogs with greater aggressivity showed a higher differential response to

22 the reward signal versus no-reward signal presented by the unfamiliar human and

23 computer, while dogs with lower aggressivity showed a higher differential response

24 to the reward signal versus no-reward signal from their handler. This suggests that 25 specific facets of canine temperament bear more strongly on the perceived reward 26 value of relevant communication signals than does reinforcement history, as each of 27 the dogs were reinforced similarly for each signal, regardless of the source (familiar 28 human, unfamiliar human, or computer). A group-level psychophysiological

29 interaction (PPI) connectivity analysis showed increased functional coupling between

30 the caudate and a region of cortex associated with visual discrimination and learning

31 on reward versus no-reward trials. Our findings emphasize the sensitivity of the

32 domestic dog to human social interaction, and may have other implications and

33 applications pertinent to the training and assessment of working and pet dogs.

\section{INTRODUCTION}


35 The domestic dog is a rising star in behavioral neuroscience due both to his high 36 trainability and likely co-evolution with humans over the last 10,000-30,000 years.

37 Recently, dogs have even proved amenable to participation in awake, unrestrained 38 neuroimaging, allowing researchers to further probe the workings of the canine mind 39 in a non-invasive and ethical method (Berns, Brooks \& Spivak, 2012). The picture 40 that is emerging suggests dogs are highly sensitive to social context and cues, both 41 from other dogs and from humans. Unsurprisingly for a highly social species, dogs 42 use an extensive and nuanced vocabulary of cues in both affiliative and antagonistic 43 interactions with conspecifics (Bradshaw \& Nott, 1995; Quaranta, Siniscalchi \& 44 Vallortigara, 2007; Horowitz, 2009; Siniscalchi, Lusito, Vallortigara \& Quaranta, 45 2013); they are perhaps more unique in their apparent interspecies sociality 46 (Siniscalchi, Sasso, Pepe, Vallortigara \& Quaranta, 2010), showing sensitivity to 47 human cues as well. It has even been suggested that dogs might serve as better 48 comparative models for human cognition than non-human apes (Topal, Miklosi, 49 Gacsci, et al., 2009). They can perform fast mapping of novel words (Kaminski, Call 50 \& Fischer, 2004), and appear to have brain regions specialized for processing the 51 human voice (Andics, Gacsci, Farago, Kis \& Miklosi, 2014). Behavioral work shows 52 that dogs can read naturalistic signals from humans that may be difficult for other species (Hare \& Tomasello, 2005; Gergely, Kupan, Miklosi \& Topal, 2012; Gacsi, Vas, 54 Topal \& Miklosi, 2013) (although see (Udell, Dorey \& Wynne, 2010)). Prior fMRI 55 research in our lab has shown that the ventral caudate nucleus-a brain region 56 known to be specialized for reward prediction and processing of positively valenced 57 stimuli across species (Schultz, Tremblay \& Holleman, 2000; Montague \& Berns, 58 2002; Humphries \& Prescott, 2010; Daw, Gershman, Seymour, Dayan \& Dolan, 59 2011)-is differentially active in dogs in response to the scent of familiar humans 60 versus familiar dogs and unfamiliar humans (Berns, Brooks \& Spivak, 2014). In line 61 with behavioral evidence (Miklosi \& Topal, 2013), this suggests that, in a real sense, 62 dogs may prefer the company of familiar humans over the company of either 63 familiar or unfamiliar dogs.

64 While the amassing data are suggestive of specialized social sensitivity in the dog, 65 the question remains to what extent simple conditioning and reinforcement history, 66 as opposed to social specialization, can explain previous findings. To address one 67 aspect of this question, we iterated on and expanded our earlier fMRI work with 68 dogs. Having previously shown a replicable but heterogeneous ventral caudate 69 response in dogs to signals from their handlers predicting either incipient food 
70 reward or no reward (Berns, Brooks \& Spivak, 2012; Berns, Brooks \& Spivak, 2013),

71 here we used fMRI to examine neural responses to these same signals presented by

72 a familiar human and an unfamiliar human and to analogous but illustrated hand

73 signals projected on a screen (referred to forthwith as "computer" signals). Although

74 the subject dogs had an extensive reinforcement history with the human and

75 computer signals, we hypothesized that differential caudate BOLD response in the

76 reward versus no-reward condition-taken here to be indicative of strength of reward

77 prediction-would be highest with the familiar human source, and lowest with the

78 computer, in line with the possibility that social bond, and not just food-specific

79 reinforcement history, affects the valence of familiar cues. There is growing

80 evidence to suggest that human-dog social bonds are durable and complex, showing

81 much of the same nuance as those of human-human social bonds (e.g., Prato-

82 Previde, Custance, Spiezio \& Sabatini, 2003; Siniscalchi, Stipo \& Quaranta, 2013). It

83 is possible then that the source of a signal might indeed have a profound effect on

84 how it is received and processed by a dog.

85 To examine possible effects of temperament on conditional neural response, we also

86 collected CBARQ questionnaires (a validated method for quantifying dog

87 temperament (Duffy \& Serpell, 2012)) from each dog's primary handler.

88 Finally, we conducted an exploratory psychophysiological interaction (PPI) analysis

89 on the BOLD data. PPI is a connectivity measure that allows one to examine what

90 brain areas increase functional connectivity (i.e., synchronous fluctuations of brain

91 activity (Biswal, Yetkin, Haughton \& Hyde, 1995)) with a seed region on a task or

92 condition-specific basis. While typical BOLD contrasts used in fMRI tend to highlight

93 regions maximally specific to the task or condition of interest, PPI can highlight more

94 distributed network activity involving areas less specialized to the primary task

95 (Friston, Buechel, Fink, Morris, Rolls \& Dolan, 1997; Rogers, Morgan, Newton \& Gore,

96 2007). PPI generally requires large numbers of events, so we collapsed across all

97 source conditions (familiar human, unfamiliar human, and computer) and looked for

98 areas with increased functional connectivity to the ventral caudate in the reward vs

99 no-reward conditions. This opens the possibility of uncovering task-specific reward

100 networks across the canine brain.

\section{MATERIALS AND METHODS}




\section{Participants}

103 Participants were dogs $(n=13)$ from the Atlanta community (Table 1$)$. All were pets

104 and/or released service dogs whose owners volunteer their time for fMRI training

105 and experiments. All had previously completed an fMRI session in which two hand

106 signals were presented by their primary trainer, one indicating forthcoming food

107 reward, the other indicating no reward. Accordingly, all dogs had demonstrated an

108 ability to remain still during training and scanning for periods of 30 seconds or

109 greater. However, one dog exhibited excessive motion during this experiment and

110 was subsequently excluded from analysis because of insufficient observations after

111 motion censoring (see below).

112 This study was performed in strict accordance with the recommendations in the 113 Guide for the Care and Use of Laboratory Animals of the National Institutes of 114 Health. The study was approved by the Emory University IACUC (Protocol \#DAR115 2001274-120814BA), and all dogs' owners gave written consent for participation in 116 the study.

\section{Training}

118 For participation in previous experiments (details in: Berns, Brooks \& Spivak, 2013; 119 Berns, Brooks \& Spivak, 2014), dogs took part in a training program using behavior 120 shaping, desensitization, habituation, and behavior chaining to prepare them to be

121 comfortable with the physical confines of the MRI bore and the loud noise produced 122 by scanning. The end goal of initial training was that each dog remain comfortable

123 and motionless for up to 30 seconds at a time during live scanning.

124 In the current experiment, dogs received reward and no reward signals from a

125 familiar human (their primary experimental trainer), an unfamiliar human, and from

126 computer-generated stimuli on a projection screen. As mentioned above, each dog

127 in this experiment was highly trained on two hand signals, an upright raised left

128 hand signifying reward, and both hands held sideways and inward-pointing, with the 129 back of each hand toward the dog, signifying no reward (as described in Berns,

130 Brooks \& Spivak, 2012). No further training for these signals was required.

131 The current experiment involved two new experimental stimuli, computer-generated

132 images to be presented on a projection screen. These were to serve the same 
133 signaling function as the previously learned hand signals (reward and no-reward).

134 The computer reward signal consisted of an illustrated image of a raised hand,

135 oriented so it would appear as a raised left hand facing the dog. The computer no-

136 reward signal consisted of two illustrated inward pointing hands with thumbs up,

137 oriented to appear as if the backs of the hands were facing the dog (Figure 1).

138 For maximum discriminability, the two signals were presented on black

139 backgrounds, and each at one of the wavelengths to which dogs are most sensitive

140 (Neitz, Geist \& Jacobs, 1989), a yellow-green for the reward signal (555 nm), and a

141 medium blue $(429 \mathrm{~nm}$ ) for the no-reward signal. Wavelengths were converted RGB

142 values (http://rohanhill.com/tools/WaveToRGB/). Because we were not making

143 predictions about visual processing, but rather wanted two stimuli that were

144 maximally discriminable, we did not attempt to normalize the contrast and

145 luminance of the two computer stimuli.

146 Training for the current experiment involved biweekly instruction at our training

147 facility overseen by core project staff and regular training at home overseen by each

148 dogs' primary experimental trainer. Dogs were trained to associate the green raised

149 hand signal with reward and the sideways blue hand signal with no reward via the

150 same method previously employed to teach them the human hand signals (Berns,

151 Brooks \& Spivak, 2013). During training sessions, each dog cooperatively stationed

152 him- or herself in a custom-made foam chinrest placed inside of a mock MRI coil.

153 Presentation of signals was contingent on the dog's being able to station calmly and

154 with minimal motion. The signals were displayed on a laptop screen placed

155 approximately 20 inches in front of the dog, and were presented in short ( $<20$ trials)

156 sessions. Presentation order was semi-randomized (each signal was presented no

157 more than 4 times consecutively, and reward to no-reward ratio was between 1:1

158 and 1:3, with the reward signal becoming less frequent once each dog became

159 comfortable and experienced with the protocol). If the dog was able to hold still for

160 the duration of the green reward hand signal, a food reward was dispensed

161 immediately after. Following the blue sideways hand signal, no reward was

162 dispensed. If the dog left the station during either signal, the stimulus was turned

163 off, and the handler waited until the dog returned, at which point they presented the

164 same stimulus again. 
165 Association learning with the two computer stimuli was conducted over multiple

166 sessions and for an amount of time comparable to initial training with the human

167 hand signals (approximately 1 month).

168 When dogs were accustomed to viewing the computer signals, further training was 169 conducted at our training facility. First, dogs were acclimated to a new food-delivery

170 system (dubbed the "treat-kabob"). This was necessary because, while previously

171 food reward had been delivered directly by hand, in the current experiment the

172 projection screen required for presentation of the computer stimuli made this

173 difficult. Furthermore, we wished to evaluate only the context variability of the

174 signals. Therefore, we needed to keep the food delivery methodology constant

175 across the three signal source states. Two variants of the food-delivery system were

176 used-one in which a long wooden dowel with a small treat placed on a dull skewer

177 at the end was slid up a PVC pipe and presented to the dog, and another in which

178 the same dowel was used to bring the treat to the dog's mouth without the PVC

179 guide tube (the specific delivery method was adjusted for the dog's comfort). In both

180 cases, dogs were first exposed to the treat kabob in their chin stations on the ground

181 until it was determined they were not nervous and were competent at removing the

182 treats from the skewer. Training was then transitioned into the mock MRI scanner

183 (Figure 2a, see Berns, Brooks \& Spivak, 2013). In 1-3 sessions with each dog's

184 primary experimental trainer presenting the familiar reward and no-reward hand

185 signals, food reward was delivered via the treat kabob by a project staff member

186 sitting below and to the side of the bore. Each dog also received one or two sessions

187 with an unfamiliar human delivering the reward and no-reward hand signals, to

188 assess their willingness to remain stationary in the presence of an unfamiliar human.

189 Note that the human used in this behavioral assessment was different than the

190 unfamiliar human who delivered signals at testing. When dogs were judged

191 comfortable with this setup, training commenced for acclimation to a screen being

192 placed in the bore. A computer monitor was placed at the end of the bore, directly in

193 front of the dog's chin station, to simulate the projection screen used in live

194 scanning. These training sessions were aimed at making the dogs comfortable with

195 stationing in the bore with a visual barrier in front of them.

196 We did not have a strict behavioral metric to determine when this training was

197 complete-rather a practical measure was used. Dogs were cleared for testing when

198 they had completed at least one month of regular practice and were able to do an 
199 extended "dress rehearsal" session in the mock scanner without showing anxiety or

200 escape behavior. This session involved stationing with the monitor in place, recorded

201 scanner noise playing, and the primary handler out of view. Dogs also had to be

202 wearing their ear protection, and treats were delivered via the treat kabob. Total

203 time in training, from first introduction to the computer stimuli to finishing "dress

204 rehearsal" ranged from 8 weeks to 16 weeks, with the duration affected by both the

205 aptitude of the dog and the diligence of the owner in implementing homework

206 sessions.

207 MRI Scanning

208 All scanning for the current experiment was conducted with a Siemens 3 T Trio 209 whole-body scanner. Dogs were stationed in their custom chin-rests, which had been 210 placed in a standard neck coil as previously described in Berns et al., 2013 (figure 211 2b). All participants wore ear protection during scanning, either Mutt Muffs ${ }^{\mathrm{TM}}$ or ear 212 plugs with wrap, depending on dog and owner preference.

213 Esrh scan session began with a 3s, single image localizer in the sagittal plane (SPGR 214 sequence, slice thickness $=4 \mathrm{~mm}, T R=9.2 \mathrm{~ms}, T E=4.15 \mathrm{~ms}$, flip angle $=40$ degrees, $215256 \times 256$ matrix, FOV=220mm).

216 A T2-weighted structural image was previously acquired during one of our earlier 217 experiments using a turbo spin-echo sequence (25-30 $2 \mathrm{~mm}$ slices, TR=3940 ms, $218 \mathrm{TE}=8.9 \mathrm{~ms}$, flip angle $=131$ degrees, 26 echo trains, $128 \times 128$ matrix, FOV $=192 \mathrm{~mm}$ ), 219 which lasted $\sim 30 \mathrm{~s}$.

220 Functional scans used a single-shot echo-planar imaging (EPI) sequence to acquire 221 volumes of 24 sequential $3 \mathrm{~mm}$ slices with a $10 \%$ gap ( $T E=28 \mathrm{~ms}, \mathrm{TR}=1400 \mathrm{~ms}$, flip 222 angle=70 degrees, 64x64 matrix, $3 \mathrm{~mm}$ in-plane voxel size, FOV=192mm). Slices 223 were oriented dorsally to the dog's brain (coronal to the magnet, as, in the sphinx 224 position, the dogs' heads were positioned 90 degrees from the usual human 225 orientation) with the phase-encoding direction right-to-left. Sequential slices were 226 used to minimize between-plane offsets from participant movement, and the $10 \%$

227 slice gap minimized the crosstalk that can occur with sequential scan sequences.

228 Six runs of up to 300 functional volumes were acquired, each lasting approximately 2296 minutes. For the first dog (Zen), three runs of up to 600 functional volumes were 
230 acquired, each lasting approximately 12 minutes. This was subsequently split into 6

231 runs in a counterbalanced fashion for the remainder of the dogs. As part of a

232 separate experiment, an additional functional run was acquired during the session in

233 which the dog was presented with different types of visual stimuli on the screen;

234 however, these data are not analyzed or reported here.

235 During functional scanning, reward was delivered via the aforementioned treat236 kabob, operated by a project staff member sitting below and to the side of the 237 scanner bore (out of sight of the dog).

\section{Experimental Design}

239 Stimuli for this experiment were as described above: the two natural hand signals 240 representing reward and no-reward delivered by a familiar and an unfamiliar human,

241 and the two illustrated computer signals representing reward and no-reward,

242 projected on a screen at the head of the bore.

243 Each subject dog received 15 reward and 15 no-reward signals with the familiar 244 human, the unfamiliar human, and with the computer images, for 90 trials overall 245 across the three source conditions. Each stimulus was presented for approximately

24610 seconds, regardless of source. These 90 trials were broken into six runs of 15

247 trials each. An event-based design was used, with reward and no-reward trials

248 presented semi-randomly within each run (either 7 reward trial and 8 no-reward

249 trials or vice versa, and with no more than three of either stimulus type presented

250 consecutively). The six runs for each dog were always in the same order: familiar

251 human, unfamiliar human, computer, computer, unfamiliar human, familiar human

252 (as part of a different experiment, an additional run was included in the middle of

253 this sequence in which different visual stimuli were presented on the computer

254 screen and are analyzed and reported elsewhere). Low sample size didn't allow for

255 different counterbalancing across participants or randomizing order, and running

256 each source condition on a separate day to avoid effects from in-session habituation

257 was not practical. This ABCCBA pattern of presentation controlled, at least partially,

258 for effects in the BOLD signal from habituation, sensitization, and scanner drift

259 becoming confounded with signal source. In addition, we determined that placing

260 the computer runs first or last might be difficult for some dogs-in practice we had

261 observed that dogs tended to move more and show more signs of anxiety when 
262 receiving computer signals as opposed to receiving signals directly from their

263 handlers. By placing the computer signals in the middle of the experimental

264 sequence, each dog had a chance to "warm up" to the task with the more familiar

265 source conditions before the computer runs, but also had the more familiar

266 (potentially easier) source conditions at the end of the experimental sequence, when

267 stress and fatigue may have made them less inclined to continue participating. The

268 first dog's 90 trials were broken instead into three runs of 30 trials each ( 15 reward

269 and 15 no-reward), in the following order: familiar, unfamiliar, computer. Thus, he

270 received the same amount of total trials and trials for each condition as the other

271 dogs, but without the pyramidal order provided the other dogs.

272 Behavioral criteria during testing for all dogs were the same as during training-

273 each dog was required to hold still for the duration of each signal. Following a

274 reward signal, they received a small piece of hot-dog via the treat-kabob. No reward

275 was given following a no-reward signal. Following each 15-trial run, the dog was

276 taken out of the scanner and allowed to walk around, drink water, etc.

277 For live scanning, some dogs did show some anxiety when first placed in the bore

278 with the projection screen in place and their handler out of sight. Basic training

279 mechanisms were employed to work around this, reprising the initial acclimation

280 approach taken in the mock scanner at our training facility discussed previously. In

281 brief, prior to the beginning of these scanner runs, the dogs were stationed with the

282 screen present and their owner's face in view. They were rewarded a number of

283 times as the owner was approximated back and to the side, and then live scanning

284 began.

\section{Event Recording}

286 Trial events (onset and offset of reward and no-reward signals) were recorded by an 287 observer via a four-button MRI-compatible button-box. The observer stood next to 288 the experimental trainer and unfamiliar person respectively on runs $1,2,5$, and 6 , 289 such that they could see the dog's head in the bore of the magnet. On these runs, 290 the observer signaled the experimental trainer and unfamiliar person when to 291 present which signal. On runs 3 and 4, the computer runs, the observer moved to 292 the side so that the dog was unable to see them, but the they could just see the 293 very tip of the dog's nose (to ascertain that the dog was still appropriately 
294 stationed/not moving). On the computer runs, the observer used the button-box to

295

296

297 A laptop computer running Matlab (MathWorks) and Cogent (FIL, University College present and advance the reward and no-reward stimuli (while simultaneously recording onset/offset).

298

299 London) was connected to the button-box via serial port, and recorded both the button-box responses by the observer and scanner sequence pulses.

\section{C-BARQ}

301 Because we hypothesized that variability in participant disposition toward handlers 302 and strangers could have a strong impact on the relative reward value of the two 303 signal types across different source conditions the dogs' handlers completed the 304 Canine Behavioral Assessment \& Research Questionnaires (C-BARQ). These have 305 been used on over 20,000 dogs and represent a standardized and validated (Duffery $306 \&$ Serpell, 2012) tool for obtaining behavioral measures from owner report. The C307 BARQ consists of 101 questions asking the respondent to report on how the dog 308 typically responds to common events.

309 Scores between 0 (minimum) and 5 (maximum) are then computed for 14

310 behavioral categories. . Because of the small number of dogs relative to the number 311 of C-BARQ factors, there was a high potential for factors to be correlated with each 312 other. This collinearity, combined with the number of factors, would be problematic 313 in any modeling of the neural data. Therefore, we performed principal component 314 analysis (PCA) on the 14 C-BARQ scores from our subjects, with a limit of four 315 factors. By limiting to four factors, we were able to include these factors in the 316 neural model without overfitting, while still being able to identify the major 317 groupings of C-BARQ dimensions that were responsible for differences in neural 318 activation.

\section{Functional Data Preprocessing and Analysis}

320 Preprocessing was conducted using AFNI (NIH) and its associated functions, and 321 most steps were identical to those listed in Berns, Brooks \& Spivak (2014). In brief, 322 2-pass, 6-parameter affine motion correction was used with a hand-selected 
323 reference volume for each dog. Because dogs moved between trials (and when

324 rewarded), aggressive censoring was carried out, relying on a combination of outlier

325 voxels in terms of signal intensity and estimated motion. Censored files were

326 inspected visually to be certain that bad volumes (e.g., when the dog's head was

327 out of the scanner) were not included. The majority of censored volumes followed

328 the consumption of food. On average, $51 \%$ of total EPI volumes were retained for

329 each subject (ranging from 38\% to 60\%). This was in line with previous experiments

330 using reward and no-reward signals (Berns, Brooks \& Spivak, 2013; Berns, Brooks \&

331 Spivak, 2014). In addition, we computed the scan-to-scan movement from the AFNI

332 motion files in all three principal directions during exposure to each source (handler,

333 stranger, computer) and condition (reward and no-reward) for each dog for all

334 volumes, including those subsequently censored. Scan-to-scan movement was

335 computed as: $\operatorname{sqrt}\left(d x_{i}{ }^{2}+d y_{i}{ }^{2}+d z_{i}{ }^{2}\right)$ where $d x_{i}, d y_{i}$, and $d z_{i}$ are the changes in

336 corresponding head position of the $i^{\text {th }}$ volume (e.g. $d x_{i}=x_{i}-x_{i-1}$ ). Scan-to-scan

337 movements greater than $10 \mathrm{~mm}$ occurred occasionally if a dog moved out of the

338 field of view. In these cases, the motion estimates were not reliable and so these

339 values were capped at $10 \mathrm{~mm}$ to avoid biasing the average. We also computed the

340 proportion of censored volumes during exposure to each source and condition. This

341 allowed us to determine whether unbalanced motion parameters or censoring

342 between reward and no-reward conditions might introduce strong bias into our BOLD

343 findings.

344 EPI images were smoothed and normalized to \%-signal change. Smoothing was

345 applied using 3dmerge, with a $6 \mathrm{~mm}$ kernel at Full-Width Half-Maximum (FWHM). The

346 resulting images were then input into the General Linear Model.

347 For each subject, a General Linear Model was estimated for each voxel using

348 3dDeconvolve. The task-related regressors in this model were: (1) familiar human

349 reward signal, (2) familiar human no-reward signal, (3) unfamiliar human reward

350 signal, (4) unfamiliar human no-reward signal, (5) computer reward signal, and (6)

351 computer no-reward signal. Because our previous work measuring the

352 hemodynamic response function (hrf) in dogs on this task revealed a peak response

353 at 4-6 s after signal onset (Berns, Brooks \& Spivak, 2012), the six task regressors

354 were modeled as impulse functions. Events were convolved with a single gamma

355 function approximating the hrf. Motion regressors generated by the motion

356 correction were also included in the model to further control for motion effects. A 
357 constant and linear drift term was included for each run. Finally, to control for 358 possibly confounding physiological factors (e.g., if the reward condition led to more 359 rapid breathing, which might boost neural BOLD signal), a spherical ROI ( $3 \mathrm{~mm}$ 360 radius) was drawn manually on each dog's structural image in the posterior 361 ventricle, just posterior to the splenium of the corpus callosum. Using AFNI's 362 3dmaskave, average timecourses for these ROIs were then extracted after 363 transforming to each dog's structural space. This timecourse was entered into the 364 general model as a nuisance variable for each subject. Because BOLD signal 365 changes in CSF and white matter reflect physiological effects, not neural processing, 366 this controlled for undue influence of physiological effects on the primary contrasts 367 (Weissenbacher, Kasess, Gerstl, Lancenberger, Moser \& Windischberger, 2009; 368 Murphy, Birn \& Bandettini, 2013).

\section{ROls and Mixed-Effects Modeling}

370 To measure the interaction of reward and no-reward signals with signal source, we 371 used a mixed-effects ANOVA to compare mean caudate activation in the six 372 conditions ( 2 signals $\times 3$ sources) across all dogs. The four primary temperament 373 factors (as computed from the CBARQ behavioral questionnaire and subsequent PCA

374 discussed above) were also included in the statistical model. To ensure that we were, 375 in fact, measuring caudate activity, we used anatomically defined ROIs. A left and 376 right ROI was drawn on each dog's structural image (Figure 3). Then, these ROIs 377 were used to extract average beta values for each dog in each condition from the 378 first-level GLMs, after transforming to each dog's structural space. The end result 379 was 12 values for each dog (the 6 conditions for both left and right caudates).

380 Analysis was conducted using the Mixed Models procedure in SPSS v21 (IBM). A $2 \times 3$ 381 ANOVA was formulated with fixed effects for hand signal (reward, no reward) and 382 source (familiar, unfamiliar, computer) with dog as a random effect. Because no 383 significant left/right differences were observed, side was not included as a fixed 384 effect. We also conducted a second analysis that included the C-BARQ temperament 385 factors as well their interactions with the other fixed effects. This allowed us to 386 determine the relative caudate activation in each of the six conditions and examine 387 how this might be modulated by temperament. 
389 In addition, we used our data to compute a psychophysiological interaction (PPI)

390 analysis at the individual and group levels. PPI highlights areas that increase

391 functional coupling (defined as synchronous firing patterns) with a seed area in a

392 certain condition, or in a certain condition in contrast to some other (Friston,

393 Buechel, Fink et al., 1997), and has been shown to produce reliable and robust

394 measures of task-specific functional connectivity (Kim \& Horwitz, 2008). PPI allows

395 one to determine which brain areas increase functional connectivity with an area of

396 interest during processing of a specific task.

397 We computed our PPI in AFNI as described in

398 (http://afni.nimh.nih.gov/sscc/gangc/CD-CorrAna.html). First, functionally localized,

399 bilateral caudate seeds were generated for each dog from the allrew-allnorew

400 contrast (that is, across all three source conditions: familiar, unfamiliar, and

401 computer). For each animal, the seeds were generated as spheres with a $3 \mathrm{~mm}$

402 radius centered on the voxel with the greatest differential activation in the left and

403 right caudate respectively.

404 The activation time series for the left and right caudate seeds were extracted for

405 each subject using 3dmaskave. The time series were then deconvolved using

406 3dDetrend using a basic gamma function. Next, the interaction of the condition

407 times (reward, no reward, and neutral baseline) and the deconvolved caudate seed

408 time series was computed to produce an interaction term. The interaction term was

409 convolved using the AFNI waver command with a basic gamma function. The

410 convolved interaction term and the initial extracted caudate seed time series were

411 then entered as regressors into the initial GLM (along with the primary task

412 regressors, drift terms, motion regressors), and physiological regressor).

413 Increased BOLD activation corresponding to the interaction term in the GLM should

414 then be seen in areas that increased functional coupling (i.e., synchronous firing)

415 with the caudate during reward versus no-reward conditions. Such areas may be

416 understood to be differentially connected to the caudate during processing of the

417 reward signal in this task. Note that, as a connectivity analysis, PPI is driven by

418 patterns of increase and decrease in BOLD signal, and is not necessarily indicative of

419 a main effect difference in BOLD strength in any particular region. 
421 To apply the transformations to a statistical contrast, the appropriate individual-level

422 contrast (obtained from the GLM as described above) was extracted from the AFNI

423 BRIK file and normalized to template space. Group normalization was conducted

424 using the Advanced Normalization Tools (ANTs) software, as described in Berns,

425 Brooks \& Spivak (2014). Briefly, three spatial transformations were computed for

426 each dog: (1) rigid-body mean EPI to high-resolution structural (6 dof); (2) affine

427 structural to template (12 dof); and (3) diffeomorphic structural to template. Spatial

428 transformations were then concatenated and applied to individual contrasts

429 obtained from the above-described GLM model. This allowed the computation of

430 group level statistics. For group-level statistics, a high-resolution MRI beagle brain

431 atlas was used as the template (Datta, Lee, Duda et al., 2012). AFNI's 3dttest++

432 was then used to compute a t-test across dogs with the null hypothesis that each

433 voxel had a mean value of zero. All twelve dogs were used in this group analysis.

434 The group contrast we conducted was reward - no reward (across all source

435 conditions), computed as the contrast [(Rew $\left.\left.{ }_{\text {fam }}+\operatorname{Rew}_{\mathrm{unf}}+\operatorname{Rew}_{\mathrm{com}}\right)\right]-\left[\left(\right.\right.$ noRew $_{\mathrm{fam}}+$

436 noRew $_{\text {unf }}+$ noRew $_{\text {com }}$ )]. The same approach was taken to compute the group PPI

437 results. Instead of a contrast, the beta values for the interaction term (computed

438 between stimulus presentation schedule and caudate time series) from each

439 individual dog were entered into a second-level model using 3dttest++. For both the

440 primary GLM and the PPI analysis, we then calculated the average smoothness of

441 the residuals using 3dFWHMx and then used 3dClustsim to estimate the significance

442 of different cluster sizes across the whole brain after correcting for familywise error

443 (FWE).

\section{RESULTS}

445 Mean scan-to-scan movement across all sources (familiar and unfamiliar human and 446 computer) and signals (reward and no-reward) was $0.94 \mathrm{~mm}$. In a repeated

447 measures ANOVA, source was a significant predictor of motion $(F(2)=44.6, p<$

448 0.001), with motion lower in the familiar handler source than the other two. Signal,

449 however, was not a significant predictor of motion $(F(1)=0.9, p=0.366)$, nor was

450 the interaction of source and signal $(F(2)=0.08, p=0.928)$. This paralleled the

451 findings from a repeated measures ANOVA examining the percentage of volumes 
452 censored across all source and signal conditions. In the latter, source was a 453 significant predictor of motion $(F(2)=16.2, p<0.001)$, while signal was not $(F(1)=$ $4541.0, p=0.333$, nor was the interaction of source and signal $(F(2)=2.0, p=0.156)$.

455 Whole-brain group analysis of [reward - no-reward] hand signals collapsed across all 456 three source conditions (familiar human, unfamiliar human, and computer) yielded 457 robust and significant bilateral ventral caudate activation. With a single-voxel 458 significance of 0.005 , the cluster, which corrected FWE across the whole brain, was $459 \mathrm{p}=0.01$. (Figure 4).

460 In the four-factor PCA of the 14 temperament factors from the C-BARQ questionnaire 461 (Table 2), the first factor accounted for $31.1 \%$ of variance, the second for $17.5 \%$, the 462 third for $15.0 \%$ and the fourth for $13.3 \%$ of variance (accounting cumulatively for $46376.9 \%$ of total variance ). Of note, the first factor appeared to strongly represent 464 aggressivity, while the second was associated with attachment and separation.

465 In the mixed-effects ANOVA model without temperament included, the interaction of 466 source and signal was not a significant predictor of caudate activation $(F(2,67.7)=$ $4671.619, p=0.206)$. However, in the mixed-effects ANOVA model with the four 468 temperament factors included, signal was a significant predictor $(F(1,88.6)=19.3$, $469 \mathrm{p}<0.001)$, the interaction of source and signal was a significant predictor $(F(2$, $47061.8)=3.6, p<0.05$ ), the interaction of source and C-BARQ factor 1 was a 471 significant predictor $(F(2,61.8)=11.6, p<0.001)$, the interaction of source with 472 signal and C-Barq factor 1 was a significant predictor $(F(2,61.8)=29.9, p<0.001)$, 473 the interaction of source with C-BARQ factor 2 was a significant predictor $(F(2,61.8)$ $474=13.6, p<0.001)$, the interaction of source with C-BARQ factor 3 was a significant 475 predictor $(F(2,61.8)=4.9, p=0.01$, and the interaction between source, signal, and 476 C-BARQ factor 3 was a significant predictor $(F(2,61.8)=19.3, p<0.001$. Neither C-

477 BARQ factor 4, nor any of its interactions, were significant predictors of caudate 478 activation. For further analysis we focused on C-BARQ factor 1. C-BARQ factor 2 did 479 not show a significant interaction with signal, and C-BARQ factor 3 was dominated 480 by the trainability metric from the C-BARQ questionnaire. The values for our dogs 481 showed very limited range in this metric, and did not match with independent 482 assessments of actual trainability by the authors (two of whom have extensive 483 animal training experience) (Figure 5). 
484 Functional connectivity group analysis revealed bilateral clusters of increased

485 activation, in the left posterior suprasylvian region corresponding to the right

486 caudate seed, and in the right posterior suprasylvian region corresponding to the left

487 caudate seed. (Figure 6). Both clusters were significant at voxelwise $p<0.05$ (321

488 voxels for the left posterior suprasylvian region, 565 voxels for the right posterior

489 suprasylvian region), but do not survive thresholding with whole-brain corrected

490 FWE at $p<0.10$.

\section{Discussion}

492 Here we showed that, across 12 dogs, the caudate was differentially active for 493 reward vs no-reward signals when analyzed at the group level. Findings controlled 494 for confounding effects from motion, censoring, and physiological changes. This 495 builds on our previous findings showing differential caudate activation for a similar 496 task at the individual subject level (Berns, Brooks \& Spivak, 2012; Berns, Brooks \& 497 Spivak, 2013). Given this robust group finding, and the extensive literature linking 498 caudate activation to reward anticipation (Montague \& Berns, 2002; Schultz, Dayan $499 \&$ Montague, 1997; Knutson, Adams, Fong \& Hommer, 2001), the current experiment 500 provides the most definitive evidence to date that $\mathrm{FMRI}$ with unrestrained, awake 501 dogs can yield reliable and valid data. Moreover, the current results emphasize the 502 importance of the source of information to the dogs and how this interacts with their 503 temperaments.

504 Our primary interest in the current experiment was to explore the effect of signal 505 source on reward processing and the extent it matters to a dog whether a reward or 506 no-reward signal comes from a familiar or an unfamiliar human, or from a human or 507 a computer. If behavioral and neural response to reward signals are merely products 508 of specific reinforcement history, dogs should not strongly differentiate signals with 509 the same meaning across different source conditions. Differential caudate activation 510 is a reliable way to probe these questions, and can be interpreted as a marker of 511 positive salience even in the absence of a specific behavior (Ariely \& Berns, 2010;

512 Bartra, Mcguire \& Kable, 2013). Brain data now confirms prior behavioral evidence

513 indicating that dogs can generalize meaningful signals when produced by unfamiliar

514 humans, and that they can learn and respond to meaningful signals produced by 515 computers. 
516 When examined in a mixed-effects model including signal type and signal source,

517 the difference between reward and no-reward signals in the caudate was not

518 significantly different across the three sources. However, including a factor

519 representative of a key attribute of canine temperament (aggressivity) revealed

520 significant interactions between signal source and temperament. In other words,

521 signal source does matter to dogs, and apparently quite strongly-but the way in

522 which it matters is highly dependent on the dog's temperament. Specifically, dogs

523 with lower aggressivity showed a higher differential caudate response to reward

524 versus no-reward signals from their handlers, while dogs with higher aggressivity

525 showed a higher differential caudate response to reward versus no-reward signals

526 from the unfamiliar humans and the computer. It must be noted that "low" and

527 "high" aggressivity measures here are relative to our sample-none of our subjects

528 scored particularly high on C-BARQ aggressivity measures. Moreover, the differences

529 in caudate activation were not due simply to changes in physiological arousal as

530 these were controlled by the inclusion of a physiological proxy vis-à-vis an ROI in the

531 CSF. Nor were the caudate differences due to motion because there was no

532 significant difference in scan-to-scan motion of reward vs. no reward signals across

533 the 3 sources. Interestingly, the differences in caudate activation were not

534 correlated with the C-BARQ factors for attachment and separation.

535 The interrelation of individual differences and neurological and behavioral responses

536 is foundational to contemporary human psychology (e.g., Depue \& Collins, 1999;

537 Ajzen, 2005). Although less studied, there is still substantial work examining

538 temperament in non-human animals (Gosling \& John, 1999), much of it recently in

539 dogs (Jones \& Gosling 2005; Taylor \& Mills, 2006; De Meester, Pluijmakers, Vemeire

$540 \&$ Laevens, 2011; Dowling-Guyer, Marder \& D'arpino, 2011), and strong evidence

541 indicates that, just as in humans, temperament is an important factor affecting

542 neural and behavioral response in different contexts. Our current finding, that the

543 caudates of dogs with lower aggressivity respond more strongly to reward versus

544 no-reward signals from familiar handlers while those of dogs with higher aggressivity

545 respond more strongly to unfamiliar humans, is in line with prior literature on striatal

546 reward processing. Striatal response to reward depends heavily on salience (Zink,

547 Pagnoni, Martin-Skurski, Chappelow \& Berns, 2004), and anxiety predisposes one to

548 attend to possible threat (MacLeod \& Mathews, 1988). Dogs who show higher

549 aggressivity may be more aroused or more anxious in the presence of a stranger

550 than with a familiar handler, and this likely increases the salience of the unfamiliar 
551 human, and thus the striatal activation to reward. Dogs with lower aggressivity, on

552 the other hand, may find their owner relatively more salient due to prior history of

553 interaction and reward.

554 As a side note, we cannot fully rule out differences in training approaches used by

555 individual handlers as a possible contributor to our findings-it is plausible that dogs

556 with different temperament may elicit different training approaches from their

557 handlers. However, all dogs were trained to a similar criterion of task success using

558 the same general approach, with biweekly oversight by project staff, over roughly

559 the same amount of time. Because the training for this task was, at essence, very

560 simple (repeated exposures leading to classical conditioning of the reward and no

561 reward cues), it seems unlikely that slight differences in training would account for

562 the results reported here.

563 Due to our relatively large dataset (90 10-second trials per dog across all three 564 source conditions) we were also able to conduct a PPI connectivity analysis to look

565 for brain regions that increased functional coupling with the caudate at the group 566 level on reward versus no-reward trials. Functional connectivity analysis with the 567 right and left caudate seeds highlighted contralateral cortical patches (right for the 568 left caudate seed and left for the right caudate seed) in the posterior suprasylvian 569 region. There is no prior work on this region in canines, but there is a substantial 570 literature in cats, who, as carnivores, are fairly closely related to dogs. Evidence 571 suggests the posterior suprasylvian region in carnivores is a downstream visual area 572 necessary for learning and discriminating between novel visual stimuli (Markuszka, 573 1978; Updyke, 1986; Lomber, Payne \& Cornwell, 1996). The posterior suprasylvian 574 region in cats is functionally analogous to inferotemporal cortex in primates, which is 575 also shown to play an integral role in learning new visual associations, including for 576 faces (Horel, Pytko-Joiner, Voytko \& Salsbury, 1987). In addition, the posterior 577 suprasylvian region in cats and the inferotemporal region in primates have been 578 shown to share strong connections with the striatum (Yeterian \& Hoesen, 1978;

579 Royce, 1982; Webster, Bachevalier \& Ungerleider, 1993). Given this, a possible 580 interpretation of our current connectivity findings is that the caudate and posterior 581 suprasylvian regions differentially coupled contralaterally to support contextual 582 visual learning related to reward anticipation. This is particularly likely given that 583 testing occurred in a novel environment, and, in the case of the stranger source 584 condition, with a novel signal giver (likely with their own slight idiosyncrasies in 
585 signal presentation). In other words, visual features of the signal and signal

586 presentation at MRI were subtly different from those previously experienced by our

587 subjects, likely leading to additional learning about what contexts, signals, and

588 signal givers might lead to reward. Due to the fairly low statistical threshold of these

589 findings, they should be taken as descriptive and suggestive as opposed to

590 conclusive, but, due to the lack of connectivity data in canines, are still of interest.

591 In brief, we demonstrated a robust bilateral differential caudate activation to reward 592 versus no reward signals at the group level in 12 dogs across three source

593 conditions. Further, a measure of temperament, specifically aggressivity, was a 594 strong predictor of differential caudate response across the three source conditions. 595 A condition-specific functional connectivity analysis indicated increased contralateral 596 coupling between the right and left caudate and visual learning brain regions. These 597 findings provide new understanding of reward processing in the domestic dog and 598 contribute to a growing body of research on individual differences in non-human 599 animals. Particularly notable is the explanatory power of a temperament measure in 600 explaining the neural response. It is likely that there are substantial individual 601 differences in how different dogs will react across a range of contexts, and future 602 research and applied work should be sensitive to this, particularly when making 603 broad claims based on findings across a group of dogs whose temperament has not 604 been assessed. BOLD signal in the caudate may serve as a predictive measure of 605 dog temperament and amenability to different training approaches, although, due to 606 the difficulty and expense of $\mathrm{fMRI}$, application in the near term would likely be 607 restricted to special instances (e.g., assessment of military or service dogs). 


\section{Competing Interests}

609 Gregory Berns and Mark Spivak own equity in Dog Star Technologies' and developed

610 technology used in the research described in this paper. The terms of this

611 arrangement have been reviewed and approved by Emory University in accordance 612 with its conflict of interest policies.

\section{Funding Disclosure}

614 This work was funded by a grant from the Office of Naval Research (N00014-13-1615 0253).

\section{Acknowledgements}


617 We are grateful to all of the dogs' owners for the time they have devoted to training:

618 Lorraine Backer (Caylin), Cindy Keen (Jack), Patricia King (Kady), Claire Mancebo

619 (Libby), Jeff Petermann (Nelson), Cecilia Kurland (Ohana), Vicki D’Amico (Pearl),

620 Nicole Zitron (Stella), Aliza Levenson (Tigger), Lisa Tallant (Velcro), Darlene Coyne

621 (Zen), and GB's dog, Callie. Thanks to Helen Berns for developing the treat-kabob

622 and dog photos.

\section{References}

624

625

626

627

628

629

630

631

632

633

634

635

636
Ajzen, I. Personality and Behavior. (2005). Berkshire, England: Open University Press.

Ariely, D. \& Berns, G. (2010). Neuromarketing: the hope and hype of neuroimaging in business. Nature Reviews Neuroscience, 11, 284-292. doi:10.1038/nrn2795

Andics, A., Gácsi M, Faragó, T., Kis, A., Miklósi, A. (2014). Voice-sensitive regions in the dog and human brain are revealed by comparative fMRI. Current Biology, 24(5), 574-578.

Bartra, O., McGuire, J., \& Kable, J. (2013). The valuation system: A coordinatebased meta-analysis of BOLD fMRI experiments examining neural correlates of subjective value. Neuroimage, 76(1), 412-427.

Berns, G., Brooks, A., \& Spivak, M. (2012). Functional MRI in Awake Unrestrained Dogs. PloS one. DOI: 10.1371/journal.pone.0038027. 
637

638

639

640

641

642

643

644

645

646

647

648

649

650

651

652

653

654

655

656

657

658

659

660

661

662

663

664

665

666

667

668

669

670

671

672

673

674

675

676

677

678

Berns, G., Brooks, A., \& Spivak, M. (2013). Replicability and heterogeneity of awake unrestrained canine fMRI responses. PloS One. DOI: 10.1371/journal.pone.0081698

Berns, G., Brooks, A., \& Spivak, M. (2014). Scent of the familiar: An fMRI study of the canine brain responses to familiar and unfamiliar human and dog odors. Behavioural Processes. DOI: 10.1016/j.beproc.2014.02.011

Biswal, B., Yetkin, F., Haughton, V., \& Hyde, J. (1995). Functional connectivity in the motor cortex of resting human brain using echo-planar mri. Magnetic Resonance Medicine, 34(4), 537-541.

Bradshaw, J. \& Nott, H. (1995). Social and communication behavior of companion dogs. In: The Domestic Dog: Its evolution, behavior and interactions with people, ed. James Serpell. Cambridge, United Kingdom: Cambridge University Press.

Daw, N., Gershman, S., Seymour, B., Dayan, P., \& Dolan, R. (2011). Model-based influences on humans' choices and striatal prediction errors. Neuron, 69(6), 1204-1215.

Datta, R., Lee, J., Duda, J., Avants, B., Vite, C., Tseng, B., Gee, J., Aguirre, G.D, \& Aguirre, G.K. (2012). A digital atlas of the dog brain. PloS one. DOI: 10.1371/journal.pone.0052140.

De Meester, R., Pluijmakers, J., Vemeire, S., \& Laevens H. (2011). The use of the socially acceptable behavior test in the study of temperament of dogs. Journal of Veterinary Behavior: Clinical Applications and Research. 6(4), 211-224.

Depue, R. \& Collins, P. (1999). Neurobiology_of the structure of personality: Dopamine, facilitation of incentive motivation, and extraversion. Behavioral and Brain Sciences, 22(3), 491-517.

Dowling-Guyer, S., Marder, A., \& D'arpino, S. (2011). Behavioral traits detected in shelter dogs by a behavior evaluation. Applied Animal Behavior Science, 130(3-4), 107-114.

Duffy, D. \& Serpell, J. (2012). Predictive validity of a method for evaluating temperament in young guide and service dogs. Applied Animal Behaviour Science, 138(1-2), 99-109.

Friston, K., Buechel, C., Fink, G., Morris, J., Rolls, E., \& Dolan, R. (1997). Psychophysiological and modulatory interactions in neuroimaging. Neuroimage, 6(3), 218-29.

Gosling S. \& John O. (1999). Personality in nonhuman animals a cross-species review. Current Directions in Psychological Science, 8(3), 69-75.

Gácsi, M., Vas, J., Topál, J., \& Miklósi, A. (2013). Wolves do not join the dance: Sophisticated aggression control by adjusting to human social signals in dogs. Applied Animal Behaviour Science, 145(3-4), 109-122.

Hare, B. \& Tomasello, M. (2005) Human-like social skills in dogs? Trends in Cognitive Sciences, 9(9), 439-444.

PeerJ reviewing PDF | (v2014:07:2354:1:1:NEW 25 Aug 2014) 
679

680

681

682

683

684

685

686

687

688

689

690

691

692

693

694

695

696

697

698

699

700

701

702

703

704

705

706

707

708

709

710

711

712

713

714

715

716

717

718

719

Horel, J., Pytko-Joiner, D., Voytko, M., \& Salsbury, K. (1987). The performance of visual tasks while segments of the inferotemporal cortex are suppressed by cold. Behavioural Brain Research, 23, 29-42.

Horowitz, A. (2009). Attention to attention in domestic dog (Canis familiaris) dyadic play. Animal Cognition, 12, 107-118.

Humphries, M. \& Prescott, T. (2010). The ventral basal ganglia, a selection mechanism at the crossroads of space, strategy, and reward. Progress in Neurobiology, 90(4), 385-417.

Jones, A. \& Gosling S. (2005). Temperament and personality in dogs (Canis familiaris): A review and evaluation of past research. Applied Animal Behaviour Science, 95(1-2), 1-53.

Kaminski, J., Call, J., \& Fischer, J. (2004). Word learning in a domestic dog: Evidence for "fast mapping". Science, 304(5677), 1682-1683.

Kim, J. \& Hortwitz, B. (2008). Investigating the neural basis for fMRI-based functional connectivity in a blocked design: application to interregional correlations and psycho-physiological interactions. Magnetic Resonance Imaging, 26(5), 583-593.

Knutson, B., Adams, C., Fong, G., \& Hommer, D. (2001). Anticipation of increasing monetary reward selectively recruits nucleus accumbens. Journal of Neuroscience 21: RC159, 1-5.

Lomber, S., Payne, B., \& Cornwell, P. (1996). Learning and recall of form discriminations during reversible cooling deactivation of ventral-posterior suprasylvian cortex in the cat. Neurobiology, 93, 1654-1658.

MacLeod, C. \& Mathews, A. (1988). Anxiety and the allocation of attention to threat. The Quarterly Journal of Experimental Psychology Section A: Human Experimental Psychology, 40(4), 653-670.

Markuszka, J. (1978). Visual properties of neurons in the posterior suprasylvian gyrus of the cat. Experimental Neurology, 59, 146-161.

Miklosi, J. \& Topal, J. (2013). What does it take to become 'best friends'? Evolutionary changes in canine social competence. Trends in Cognitive Sciences, 17(6), 287-294.

Montague, R. \& Berns, G. (2002). Neural economics and the biological substrates of valuation

Neuron, 36(2), 265-284.

Murphy, K., Birn, R., \& Bandettini, P. (2013). Resting-state fMRI confounds and cleanup. Neuroimage, 80, 349-359.

Neitz, J., Geist, T., \& Jacobs, G. (1989). Color vision in the dog. Visual Neuroscience, 3(2), 119-125.

Rogers, B., Morgan, V., Newton, A., \& Gore, J. (2007). Assessing Functional Connectivity in the Human Brain by FMRI. Magnetic Resonance Imaging, 25(10), 1347-1357. 
749

750

751

752

753

754

755

756

757

758

759

760

761

762

763

Royce, G. (1982). Laminar origin of cortical neurons which project upon the caudate nucleus: A horseradish peroxidase investigation in the cat. Journal of Comparative Neurology, 205(1), 8-29.

Prato-Previde, E., Custance, D., Spiezio, C., \& Sabatini, F. (2003). Is the doghuman relationship an attachment bond? An observational study using Ainsworth's strange situation. Behaviour, 140(2), 225-254.

Quaranta, A., Siniscalchi, M., \& Vallortigara, G. (2007). Asymmetric tail-wagging response by dogs to different emotive stimuli. Current Biology, 17(6), R200.

Siniscalchi, M., Sasso, R., Pepe, A., Vallortigara, G., \& Quaranta, A. (2010). Dogs turn left to emotional stimuli. Behavioural Brain Research, 208(2), 516521.

Siniscalchi, M., Lusito, R., Vallortigara, G., \& Quaranta, A. (2013). Seeing left- or right-asymmetric tail wagging produces different emotional responses in dogs. Current Biology, 23(22), 2279-2282.

Siniscalchi, M., Stipo, C., \& Quaranta, A. (2013). “Like owner, like dog": Correlation between the owner's attachment profile and the owner-dog bond. PLoS One, 8(10), doi: 10.1371/journal.pone.0078455

Schultz, W., Dayan, P., \& Montague, P. (1997) A neural substrate of prediction and reward. Science, 275, 1593-1599.

Schultz, W., Tremblay, L., \& Hollerman, J. 2000. Reward processing in primate orbitofrontal cortex and basal ganglia. Cerebral Cortex, 10(2), 272-283.

Taylor, K. \& Mills, D. (2006). The development and assessment of temperament test for adult companion dogs. Journal of Veterinary Behavior: Clinical Applications and Research, 1(3), 94-108.

Teglas, E., Gergely, A., Kupan, K., Miklosi, A., \& Topal, J. (2012). Dogs' gaze following is tuned to human communicative signals. Current Biology, 22(3), 209-212.

Topal, J. Miklosi, A., Gacsi, M., Doka, A., Pongracz, P., Kubinyi, E., Viranyi, Z., \& Csany, V. (2009). The dog as a model for understanding human social behavior. Advances in the Study of Behavior, 39, 71-116.

Udell, M., Dorey, N., \& Wynne, C. (2010). What did domestication do to dogs? A new account of dogs' sensitivity to human actions. Biological Reviews, 85(2), 327-345.

Updyke, B. (1986). Retinotopic organization within the cat's posterior suprasylvian sulcus and gyrus. The Journal of Comparative Neurology, 246, 265-280.

Webster, M., Bachevalier, J., \& Ungerleider, L. (1993). Subcortical connections of inferior temporal areas TE and TEO in macaque monkeys. The Journal of Comparative Neurology, 334, 73-91.

Weissenbacher, A., Kasess, C., Gerstl, F., Lanzenberger, R., Moser, E., \& Windischberger, C. (2009). Correlations and anticorrelations in restingstate functional connectivity MRI: A quantitative comparison of preprocessing strategies. Neuroimage, 47, 1408-1416. 
764

765

766

767

768

769
Yeterian, E. \& Hoesen, G. (1978). Cortico-striate projections in the rhesus monkey: The organization of certain cortico-caudate connections. Brain Research, 139, 43-63.

Zink, C., Pagnoni, G., Martin-Skurski, M., Chappelow, J., \& Berns, G. (2004). Human striatal responses to monetary reward depend on saliency. Neuron, 42(3), 509-517. 
771 Figure 1: The computer reward (A) and no-reward (B) signals.

772 Figure 2: A. Participant Kady stationed in the mock scanner with the treat kabob. B. 773 Participant Zen stationed in the MRI bore, facing the projection screen (and wearing 774 ear protection).

775 776

777

778

779

780

781

782

783

784

785

786

787

788

789

790

791

792

Figure 3: The right caudate seed, anatomically defined, used for participant Kady, in the transverse $(A)$ and coronal $(B)$ planes.

Figure 4: Whole-brain group analysis of response to all reward - no-reward conditions. An unthresholded transverse slice $(A)$ and coronal slice $(B)$ are shown, as is a coronal slice thresholded at 0.005 (C).Color indicates t-statistic at each voxel against the null hypothesis of equal activity to reward and no-reward conditions. Significantly greater activity was observed in the reward versus no-reward condition

Figure 5: Activation within caudate ROls in dogs with low and high aggressivity (relative to our sample mean) for reward and no-reward signals across the three source conditions. Values and s.e. are derived from the full mixed-effects model, using Z-scores for C-BARQ factor 1 of +1 for dogs with higher aggressivity and-1 for dogs with lower aggressivity ( \pm 1 corresponded to the upper and lower limits of the scores). Dogs with lower aggressivity showed significantly greater caudate activation to reward versus no reward signals presented by their familiar handler $(p<0.001)$, but not from unfamiliar humans $(p=0.15)$ or computers $(p=0.09)$, while dogs with higher aggressivity showed significantly greater activation to the reward versus no reward signals from the unfamiliar human $(p=0.003)$ and computer $(p<0.001)$, but not their familiar handler $(p=0.07)$.

793

794

795

796

797

798

799

800

801
Figure 6: Whole-brain group analysis of the interaction between BOLD time course in the left and the right caudate seeds and signal presentations-warmer colors here represent increased functional coupling with the caudate seed during presentations of the reward versus no-reward signals across all three source conditions (familiar human, unfamiliar human, computer). The cluster corresponding to the right caudate seed $(A$, upper left unthresholded and $C$, lower left thresholded voxelwise at $p<0.05)$ is in the left posterior suprasylvian region, and the cluster corresponding to the left caudate seed ( $B$, upper right unthresholded and $D$, lower right thresholded voxelwise at $p<0.05$ ) is in the right posterior suprasylvian region. 
802 Color indicates $t$-statistic at each voxel against the null hypothesis of equal 803 connectivity to the caudate for reward and no-reward conditions. 
Table 1 - Participants

\begin{tabular}{|c|c|c|c|c|c|c|}
\hline Name & Breed & Sex & Age & \multicolumn{3}{|c|}{ Service training } \\
\hline Callie & Feist & Female-spayed & & 4 & $\mathrm{~N}$ & \\
\hline Caylin & Border Collie & Female-spayed & & 4 & $\mathrm{~N}$ & \\
\hline Jack & Golden Retriever & \multicolumn{3}{|c|}{ Male-neutered } & 9 & $\mathrm{~N}$ \\
\hline Kady & Yellow Lab & Female-spayed & & 3 & $\mathrm{Y}$ & \\
\hline Libby & Vizsla Pit Mix & Female-spayed & & 7 & $\mathrm{~N}$ & \\
\hline Nelson & Cairn Terrier Mix & \multicolumn{2}{|c|}{ Male-neutered } & & 3 & $\mathrm{~N}$ \\
\hline Ohana & Golden Retriever & \multicolumn{2}{|c|}{ Female-spayed } & & 4 & $Y$ \\
\hline Pearl & Golden Retriever & \multicolumn{2}{|c|}{ Female-spayed } & & 3 & $Y$ \\
\hline Stella & Bouvier & \multicolumn{2}{|c|}{ Female-spayed } & & 5 & $\mathrm{~N}$ \\
\hline Tigger & Boston Terrier & Male-neutered & & 6 & $\mathrm{~N}$ & \\
\hline Velcro & Vizsla & Male-intact & 5 & $\mathrm{~N}$ & & \\
\hline Zen & Yellow Lab & Male-neutered & & 3 & $Y$ & \\
\hline
\end{tabular}




\section{Table 2 (on next page) \\ Weighting of C-BARQ PCA Factors}


Table 2 - Weighting of C-BARQ PCA Factors

\begin{tabular}{|c|c|c|c|c|}
\hline Temperament & Componen & Componen & Componen & Compone \\
\hline Stranger-directed & $\begin{array}{l}\mathbf{t ~ 1} \\
.705\end{array}$ & $\begin{array}{l}\mathbf{t} \mathbf{2} \\
.373\end{array}$ & $\begin{array}{l}\mathbf{t} \mathbf{3} \\
-.319\end{array}$ & $\begin{array}{l}\text { nt 4 } \\
-.161\end{array}$ \\
\hline $\begin{array}{l}\text { aggression } \\
\text { Owner-directed }\end{array}$ & .882 & -.174 & -.383 & .114 \\
\hline $\begin{array}{l}\text { aggression } \\
\text { Dog-directed aggression } \\
\text { Dog-directed fear } \\
\text { Familiar-dog aggression } \\
\text { Trainability } \\
\text { Chasing } \\
\text { Stranger-directed fear } \\
\text { Nonsocial fear } \\
\text { Separation-related }\end{array}$ & $\begin{array}{l}.735 \\
.450 \\
.887 \\
-.111 \\
.785 \\
-.016 \\
.577 \\
.237\end{array}$ & $\begin{array}{l}-.104 \\
-.153 \\
-.323 \\
.043 \\
.217 \\
.028 \\
-.396 \\
.716\end{array}$ & $\begin{array}{l}.416 \\
.686 \\
.082 \\
.805 \\
.260 \\
.290 \\
.106 \\
.044\end{array}$ & $\begin{array}{l}-.209 \\
.451 \\
.123 \\
-.245 \\
.130 \\
.801 \\
-.317 \\
-.348\end{array}$ \\
\hline $\begin{array}{l}\text { problems } \\
\text { Touch sensitivity } \\
\text { Excitability } \\
\text { Attachment/attention }\end{array}$ & $\begin{array}{l}.580 \\
.341 \\
.097\end{array}$ & $\begin{array}{l}.149 \\
.613 \\
.825\end{array}$ & $\begin{array}{l}-.571 \\
.013 \\
.250\end{array}$ & $\begin{array}{l}.405 \\
-.115 \\
-.150\end{array}$ \\
\hline $\begin{array}{l}\text { seeking } \\
\text { Energy }\end{array}$ & -.270 & .587 & .016 & .654 \\
\hline
\end{tabular}




\section{Figure 1}

Figure 1

The computer reward $(\mathrm{A})$ and no-reward $(\mathrm{B})$ signals.

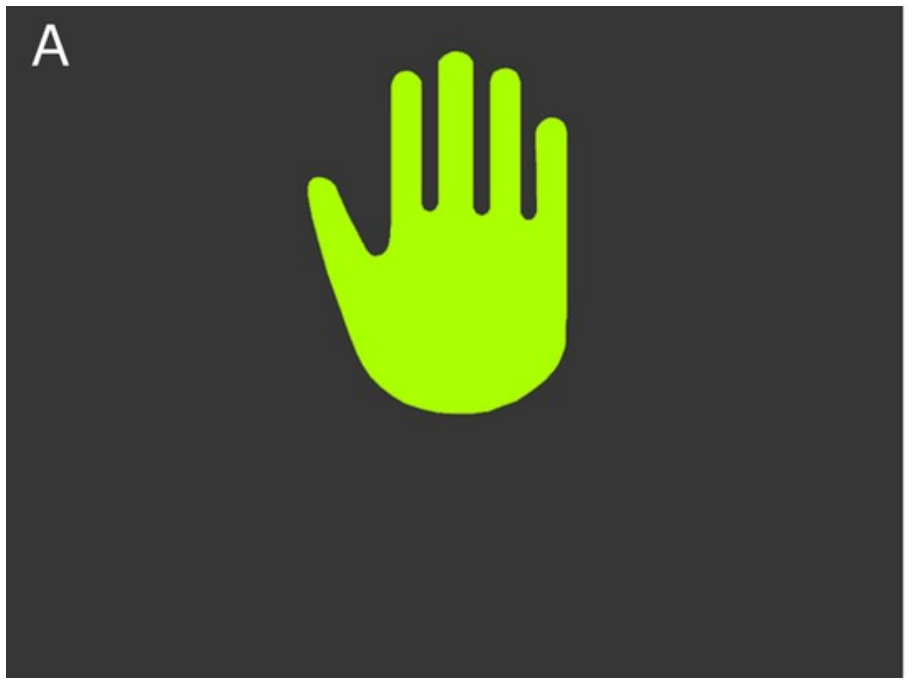




\section{Figure 2}

Figure 2

A. Participant Kady stationed in the mock scanner with the treat kabob. B. Participant Zen stationed in the MRI bore, facing the projection screen (and wearing ear protection). 


\section{Figure 3}

Figure 3

Figure 3: The right caudate seed, anatomically defined, used for participant Kady, in the transverse (A) and coronal (B) planes. 

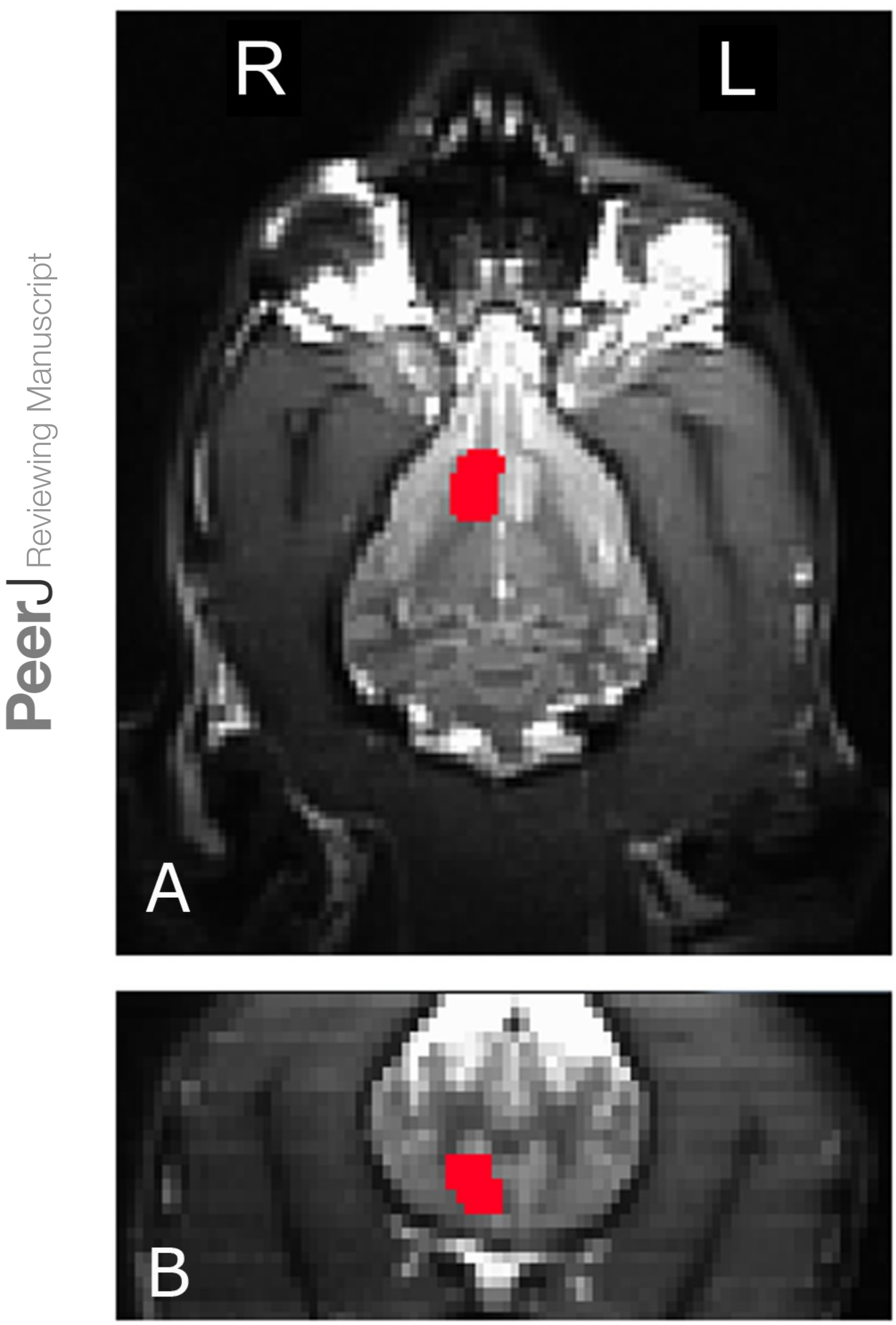

PeerJ reviewing PDF | (v2014:07:2354:1:1:NEW 25 Aug 2014) 


\section{Figure 4}

Figure 4

Figure 4: Whole-brain group analysis of response to all reward-no-reward conditions. An unthresholded transverse slice $(A)$ and coronal slice $(B)$ are shown, as is a coronal slice thresholded at 0.005 (C).Color indicates $t$-statistic at each voxel against the null hypothesis of equal activity to reward and no-reward conditions. Significantly greater activity was observed in the reward versus no-reward condition.

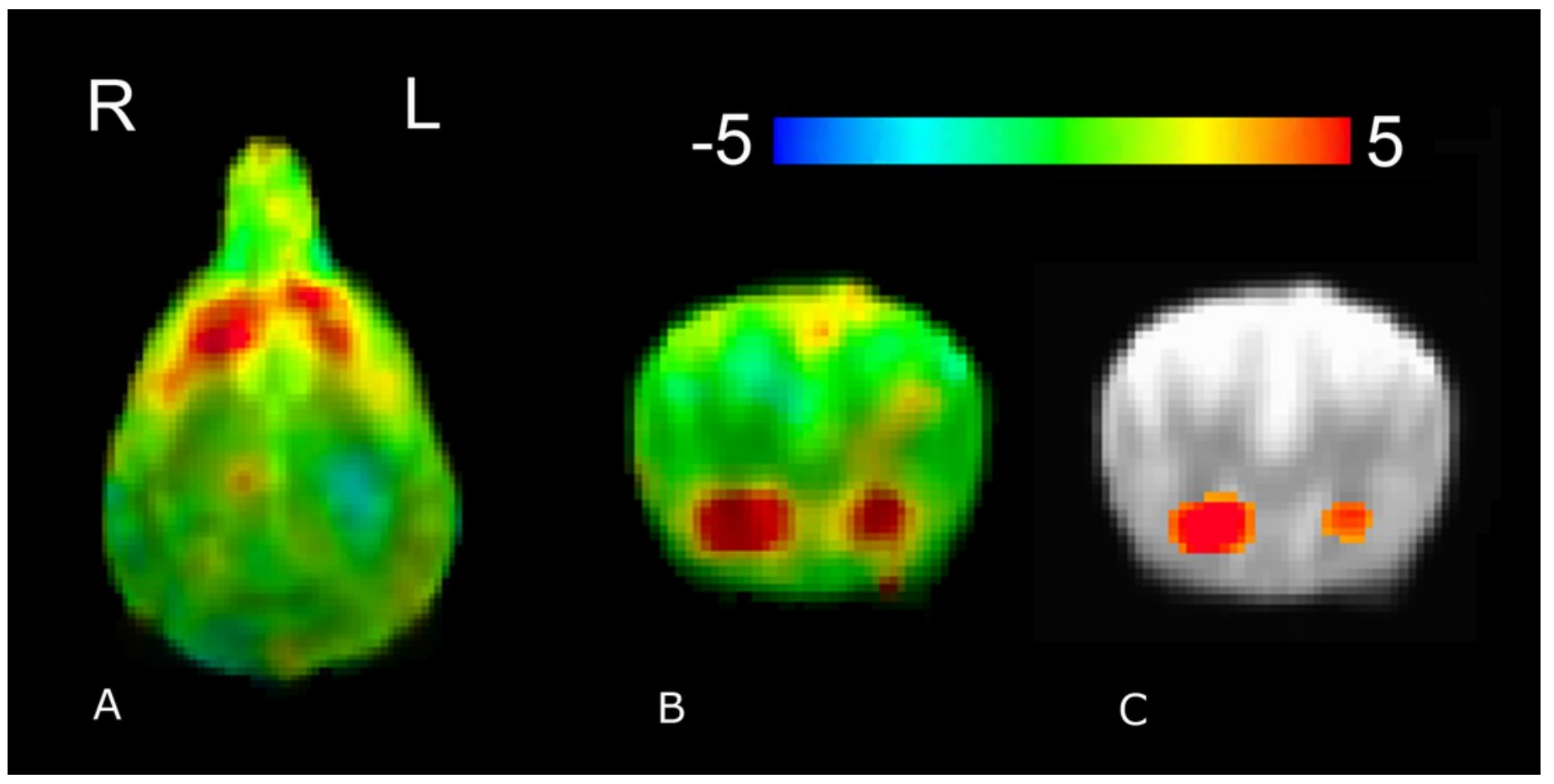




\section{Figure 5}

Figure 5

Activation within caudate ROIs in dogs with low and high aggressivity (relative to our sample mean) for reward and no-reward signals across the three source conditions. Values and s.e. are derived from the full mixed-effects model, using Z-scores for C-BARQ factor 1 of +1 for dogs with higher aggressivity and-1 for dogs with lower aggressivity $( \pm 1$ corresponded to the upper and lower limits of the scores). Dogs with lower aggressivity showed significantly greater caudate activation to reward versus no reward signals presented by their familiar handler $(p<0.001)$, but not from unfamiliar humans $(p=0.15)$ or computers $(p=0.09)$, while dogs with higher aggressivity showed significantly greater activation to the reward versus no reward signals from the unfamiliar human $(p=0.003)$ and computer $(p<0.001)$, but not their familiar handler $(p=0.07)$. 
A

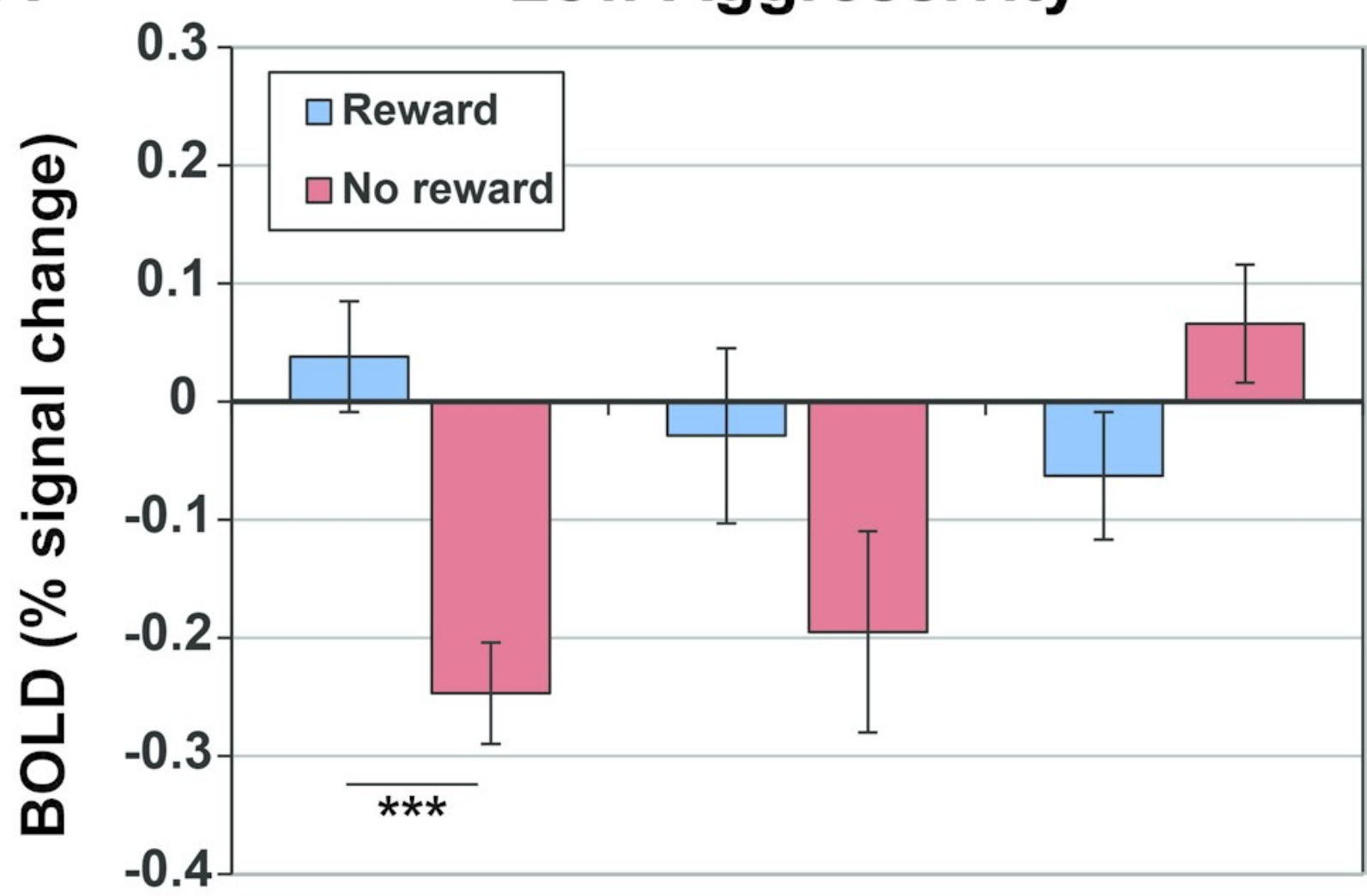

Familiar Unfamiliar Computer Human Human

B

High Aggressivity

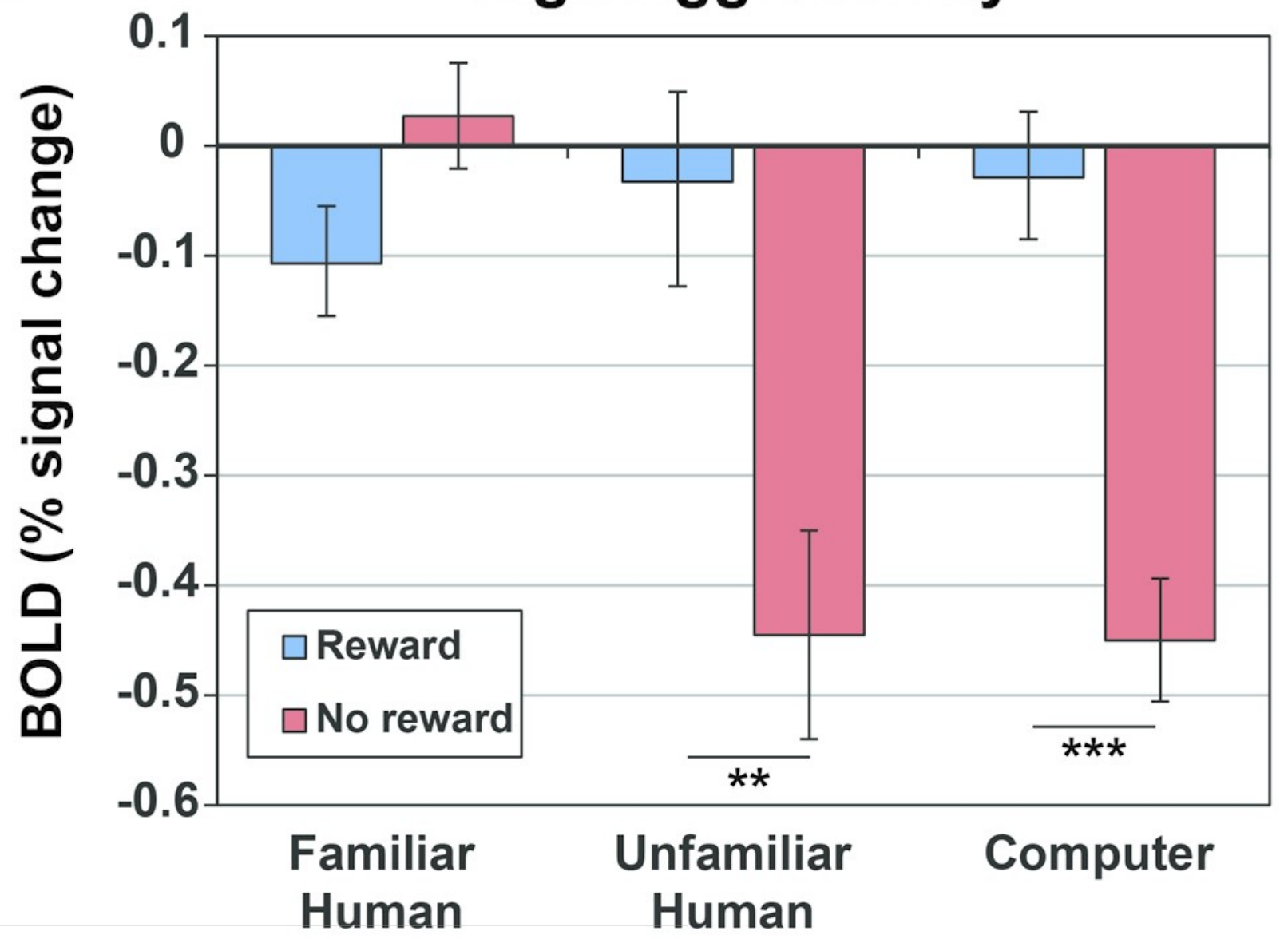




\section{Figure 6}

Figure 6

Figure 6: Whole-brain group analysis of the interaction between BOLD time course in the left and the right caudate seeds and signal presentations-warmer colors here represent increased functional coupling with the caudate seed during presentations of the reward versus no-reward signals across all three source conditions (familiar human, unfamiliar human, computer). The cluster corresponding to the right caudate seed (A, upper left unthresholded and $C$, lower left thresholded voxelwise at $p<0.05$ ) is in the left posterior suprasylvian region, and the cluster corresponding to the left caudate seed ( $B$, upper right unthresholded and $D$, lower right thresholded voxe/wise at $p<0.05$ ) is in the right posterior suprasylvian region. Color indicates $t$-statistic at each voxel against the null hypothesis of equal connectivity to the caudate for reward and no-reward conditions. 


\section{$\mathrm{R}$}

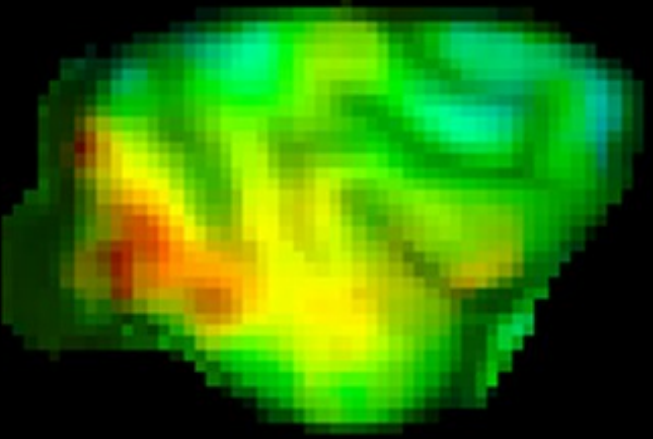

A

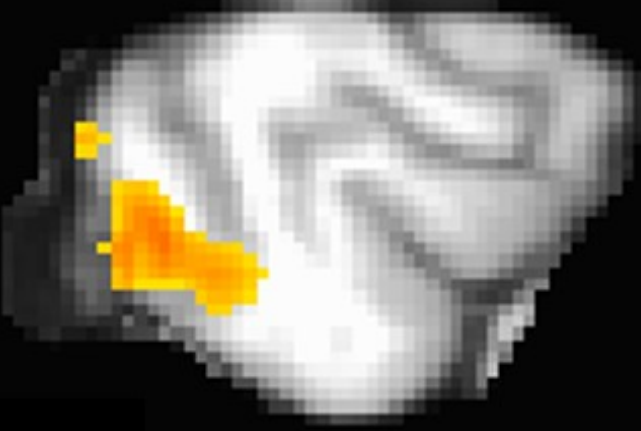

0
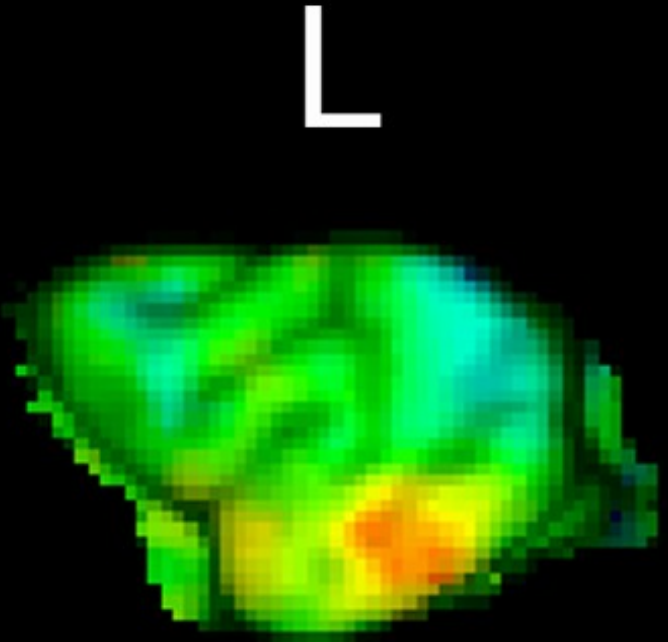

B

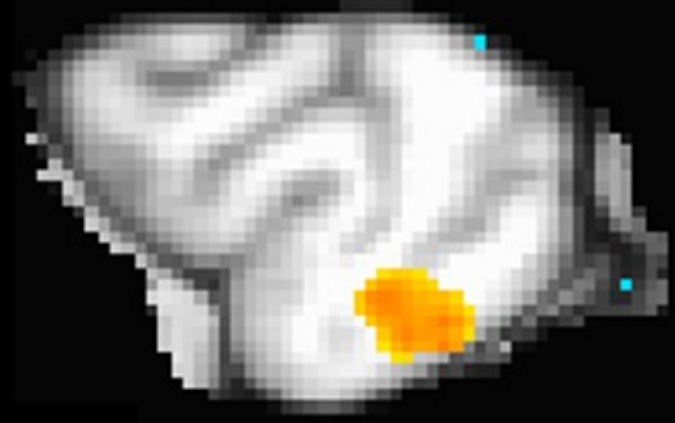

$\mathrm{D}$

$-4$ 